\title{
ATENDIMENTO ESPECIALIZADO E FORMAÇÃO PROFISSIONAL: REFLEXÕES A PARTIR DA INTERVENÇÃO EM TIMOR-LESTE
}

\section{RESUMO}

Consiste em um relato de experiência das ações de intervenção e formação profissional realizadas em uma instituição de atendimento especializado, localizada em Timor-Leste. As ações foram desenvolvidas por um grupo de quatro docentes brasileiros que atuam em um programa de cooperação educacional entre o Brasil e Timor. Este estudo apresenta o trabalho de intervenção na área da Educação Física, assim como a realização de um curso de formação profissional para funcionários do centro de reabilitação, elaborado a partir das práticas desenvolvidas pelo grupo de cooperantes. $\mathrm{O}$ atendimento especializado realizado na área da Educação Física, foi direcionado às crianças que apresentam comprometimentos físicos/motores e as atividades foram elaboradas a partir dos pressupostos metodológicos da educação psicomotora. Percebem-se algumas mudanças tanto na mobilidade das crianças atendidas, quanto no comportamento das famílias para com seus filhos. Em relação ao curso de formação, obseva-se autonomia na construção de planejamentos e nas intervenções realizadas.

Palavras-Chave: Educação especial; Educação física; Atendimento especializado; Formação profissional; Timor-leste.

\section{SPECIALIZED SERVICE AND PROFESSIONAL FORMATION: REFLECTIONS FROM THE INTERVENTION IN EAST TIMOR}

\begin{abstract}
It consists of an experience report of interventional actions and professional formation taken in an institution of specialized service, located in East Timor. The actions were developed by a group of four Brazilian teachers who act in an educational cooperative program between Brazil and Timor. This study presents the intervention work in the area of Physical Education, as well as the completion of a course at professional formation for the staff of the rehabilitation center, elaborated from the practices developed by the cooperating group. The specialized service taken in the area of Physical Education, was addressed to children who show physical/motor impairments and the activities were elaborated from the methodological presuppositions of the psychomotor education. It is perceived some changes both in the mobility of the children who were treated and in the behavior of the families with their children. In relation to the formation course for the staff, it is observed autonomy in the planning construction and in the interventions taken.
\end{abstract}

Key-Words: Special education; Physical education; Specialized service; Professional formation; East timor.

Conexões: revista da Faculdade de Educação Física da UNICAMP, Campinas, v. 13, n. 4, p. 178-187, out./dez. 2015. ISSN: 1983-9030. 


\title{
ASISTENCIA ESPECIALIZADA Y FORMACIÓN PROFESIONAL: REFLEXIONES A PARTIR DE LA INTERVENCIÓN EN TIMOR- LESTE
}

\begin{abstract}
RESUMEN
Se trata de un relato de experiencia de las acciones de intervención y formación profesional realizadas en una institución de asistencia especializada, ubicada en Timor Este. Las acciones fueron desarrolladas por un grupo de cuatro docentes brasileños que trabajan en un programa de cooperación educacional entre Brasil y Timor. Este estudio presenta el trabajo de intervención en el área de la Educación Física, así como la realización de un curso de formación profesional para los empleados del centro de rehabilitación, elaborado a partir de las prácticas desarrolladas por el grupo de cooperantes. La asistencia especializada realizada en el área de la Educación Física estuvo dirigida a niños que tienen impedimentos físicos/motrices y las actividades fueron elaboradas a partir de los presupuestos metodológicos de la educación psicomotriz. Se perciben algunos cambios tanto en la movilidad de los niños atendidos, como en el comportamiento de las familias hacia los hijos. Con relación al curso de formación, se observa autonomía en la construcción de la planificación y en las intervenciones realizadas.
\end{abstract}

Palabras-Clave: Educación especial; Educación fisica; Asistencia especializada; Formación profesional; Timor-leste.

Conexões: revista da Faculdade de Educação Física da UNICAMP, Campinas, v. 13, n. 4, p. 178-187, out./dez. 2015. ISSN: 1983-9030. 


\section{INTRODUÇÃO}

A atuação no Programa de Qualificação de Docentes e Ensino da Língua Portuguesa (PQLP/CAPES) $^{\mathrm{i}}$ possibitou algumas aproximações com as temáticas "Educação Especial” e “Educação Inclusiva” em Timor-Leste. A partir de uma solicitação do Centro de Reabilitação Limam Hamutuk, localizado no distrito de Hera (Timor-Leste), uma equipe de quatro cooperantes brasileiros passou a realizar uma intervenção sistemática com um grupo de 28 pessoas.

As ações educacionais realizadas pelos cooperantes estruturaram-se a partir de duas linhas de ação, sendo a primeira composta por intervenções com um grupo de crianças, jovens e adultos que apresentam deficiências, a fim de construir um arcabouço de vivências e reflexões sobre as possibilidades de intervenção no contexto timorense e, posteriormente, a utilização dessas intervenções para a construção e realização de um curso de formação para os funcionários do Centro de Reabilitação, sendo esta a segunda linha de ação.

Acerca do trabalho de intervenção direta com as crianças, jovens e adultos que apresentam deficiências físicas, sensoriais e cognitivas, os quatro educadores da cooperação brasileira desenvolveram o trabalho a partir de quatro distintas áreas de atuação: Oficinas de Arte e Educação, Vivências Motoras e Corporais, Práticas Pedagógicas de Inclusão Escolar e Alfabetização em Língua de Sinais. As intervenções aconteceram de junho a dezembro de 2014, uma vez por semana, das nove até o meio-dia, no Centro de Reabilitação Liman Hamutuk. O grupo, composto por 28, pessoas foi dividido em dois subgrupos, o primeiro composto por crianças e o segundo pelos jovens e adultos. Com o primeiro grupo foram desenvolvidas atividades relacionadas à alfabetização e vivências corporais e motoras. Com o segundo grupo, atividades de arte e educação. Eventualmente, ambos os grupos foram reunidos em atividades coletivas.

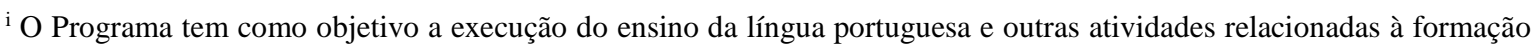
de docentes de diversos níveis das instituições de ensino timorenses (edital PQLP - 076/2013). A partir do Ajuste Complementar ao Acordo de Cooperação Educacional entre o Brasil e o Timor-Leste (BRASIL, 2011) definiu-se as áreas fundamentais do programa, em três: a) formação inicial e continuada dos docentes; b)fomento ao ensino da língua portuguesa; e c) apoio ao ensino superior. (Projeto Político Pedagógico PQLP/Capes, 2015).

Conexões: revista da Faculdade de Educação Física da UNICAMP, Campinas, v. 13, n. 4, p. 178-187, out./dez. 2015. ISSN: 1983-9030.
} 
Com relação à organização e desenvolvimento de formações para os funcionários do Centro de Reabilitação, as ações foram planejadas e ministradas coletivamente, pelos quatro cooperantes. Efetivamente, foram utilizadas na elaboração do curso de formação as experiências das diferentes intervenções realizadas pelos quatro cooperantes. O curso teve duração de dois meses, tento como objetivo apresentar noções teóricas acerca das deficiências e construir coletivamente práticas interventivas que seriam implementadas pelos formandos.

\section{CONTEXTUALIZAÇÃO}

Ações direcionadas ao atendimento especializado às pessoas que apresentam deficiências, no distrito de Hera, Timor-Leste, iniciaram no ano de 2007. De acordo com entrevistas realizadas com a administradora do centro de reabilitação, o trabalho, inicialmente assistencialista, começou a partir da ação de um grupo de voluntários cristãos, procedentes da Austrália, Nova Zelândia e do Brasil, que duas ou três vezes por semana se dirigiam até as casas das famílias e realizavam orientações sobre cuidados com a saúde e atividades para estímulo da leitura e da escrita.

O Centro de Reabilitação Limam Hamutuk surgiu, assim, da necessidade de um espaço adequado ao atendimento dessas pessoas. A partir de parcerias com instituições religiosas, mobilizou-se e viabilizaram-se recursos e mão de obra para construção do Centro de Reabilitação, que demorou cinco anos para ficar pronto. Assim, inaugurado em 2013, atualmente esta instituição especializada é mantida por recursos financeiros de parcerias com instituições religiosas e presta atendimento médico, fisioterápico e educacional à população de Hera.

Com relação ao atendimento educacional na rede regular de ensino, este é garantido por Lei no Artigo 59 da Constituição de Timor-Leste, uma vez que assegura o direito à educação e à cultura a todos os cidadãos. Contudo, só a partir da aprovação da Lei de Diretrizes e Bases da Educação em Timor-Leste, em 2008, LDB nº14/2008, ${ }^{1}$ de forma mais especifica, é assegurada às crianças com necessidades educativas especiais Conexões: revista da Faculdade de Educação Física da UNICAMP, Campinas, v. 13, n. 4, p. 178-187, out./dez. 2015. ISSN: 1983-9030. 
“designadamente, as deficiências físicas e mentais, condições adequadas ao desenvolvimento e pleno aproveitamento das suas capacidades”. Destaca-se ainda que em 2010 o Ministério da Educação de Timor-Leste realizou a primeira Conferência Nacional sobre Educação Inclusiva e afirmou, na ocasião, ser prioridade o incentivo ao atendimento às pessoas que apresentam deficiências, na rede regular de ensino.

Assim, embora haja um movimento que visa garantir o atendimento educacional das pessoas que apresentam deficiência, sabemos que, no contexto timorense, tais casos (em sua maioria) são atendidos em instituições especializadas nas comunidades de origem. Destacamos ainda que, a partir da entrevista realizada com a administradora do centro de reabilitação e da análise do contexto timorense em função da atuação do PQLP/Capes, ${ }^{2}$ podemos dizer que, considerando as fases do processo de educação inclusiva, em TimorLeste, este transita na fase da Segregação e/ou Integração. De acordo com Sassaki, ${ }^{3}$ a segregação caracteriza-se pelo atendimento realizado em instituições especializadas e, na fase da Integração, as crianças e os jovens mais aptos são encaminhados às escolas comuns, sento atendimento em classes especiais e salas de recursos.

\section{INTERVENÇÃO A PARTIR DE VIVÊNCIAS MOTORAS E CORPORAIS}

Dentre as ações de intervenção realizadas pelos docentes da cooperação brasileira, o atendimento na área da Educação Física foi denominado "Vivências Motoras e Corporais" e as atividades foram estruturadas a partir dos pressupostos metodológicos da Educação Psicomotora. Conforme Le Boulch, ${ }^{4}$ a educação psicomotora é uma metodologia que instrumentaliza o movimento humano e objetiva favorecer o desenvolvimento da criança. Segundo Chicon, ${ }^{5}$ a Educação Psicomotora é a metodologia mais indicada para possibilitar a adequação ao "fluxo de desenvolvimento dos indivíduos", uma vez que este ocorre de forma mais lenta.

Dessa forma, as atividades no Centro de Reabilitação foram realizadas de modo a criar situações que possibilitassem variadas formas de expressão e movimento, sendo este Conexões: revista da Faculdade de Educação Física da UNICAMP, Campinas, v. 13, n. 4, p. 178-187, out./dez. 2015. ISSN: 1983-9030. 
entendido como "um meio"/“um instrumento" com vistas ao desenvolvimento integral da criança. Assim, o objetivo desta ação pedagógica foi potencializar, por meio do movimento, o esquema corporal, a coordenação motora, a percepção temporal e a percepção espacial, buscando vivenciar e construir com as crianças formas de movimentos que as tornassem mais independentes.

As atividades de "Vivências Motoras e Corporais" foram realizadas a partir de duas situações distintas: a) atendimento individualizado direcionado as crianças com deficiência fisica/motora; b) atendimento em grupo para todas as crianças que frequentavam o Centro de Reabilitação. Esses atendimentos aconteceram de maneira intercalada, ou seja, em uma semana era realizado o atendimento individualizado e, na semana seguinte, o atendimento em grupo. É importante pontuar que, em ambas as situações, o trabalho foi estruturado de modo a propor situações-problema possibilitando assim a busca de soluções para esses desafios motores/corporais.

A intervenção denominada de Atendimento Individualizado foi realizada com três crianças que têm, em comum, o compromentimento fisico/motor. Uma característica dessa ação pedagógica foi a participação conjunta das mães durante a realização das atividades, uma vez que é fundamental a compreensão de que o trabalho realizado precisa ser contínuo para que haja a criação de novos hábitos corporais da criança. Dessa forma, construímos situações que exigiam a participação das mães, em função da necessidade de estimulá-las a reproduzir algumas das atividades no ambiente doméstico com a criança. Foi fundamental que elas percebessem a importância de estimular seus filhos a realizar movimentos que habitualmente não fazem devido a seus membros serem afetados pela deficiência.

A estruturação do plano de intervenção foi aplicada individualmente, seguindo uma sequência de atividades ${ }^{\mathrm{ii}}$, com o objetivo de avaliar quais seriam as capacidades motoras que poderiam ser privilegiadas nos atendimentos. Assim, a partir da análise realizada,

\footnotetext{
ii Foram realizadas atividades que avaliavam a coordenação dinâmica das mãos, coordenação dinâmica geral, equilíbrio (controle postural) e lateralidade. Foi realizado o registro da realização das atividades por meio de fotografias e vídeos.
}

Conexões: revista da Faculdade de Educação Física da UNICAMP, Campinas, v. 13, n. 4, p. 178-187, out./dez. 2015. ISSN: 1983-9030. 
consequentemente, foram priorizadas atividades que potencializam o desenvolvimento do controle postural e da lateralidade. De forma gradual, essas atividades, muitas vezes, iniciaram-se do chão para diferentes alturas e, na sequência, com a inclusão de alguns materiais como bolas de vários tamanhos e pesos, vendas para os olhos, utilização de cordas e aros de diferentes tamanhos e elásticos.

As situações de atendimento em grupo foram realizadas simultaneamente por todas as crianças que frequentam do Centro de Reabilitação. As atividades foram estruturadas a fim de que as crianças experimentassem diversas situações de jogos, brincadeiras, ou mesmo circuitos motores que potencializam a percepção do seu próprio corpo no espaço e também a interação com o meio e com os outros corpos. Algumas das atividades desenvolvidas foram: confecção de bolas de jornal, confecção de massa de modelar caseira e posterior experimentação da modelagem, atividade da “cama de gato” (circuito motor de elástico transpassado em que as crianças deveriam atravessar de uma ponta a outra de diferentes formas evitando encostar no elástico).

\section{CURSO DE FORMAÇÃO PROFISSIONAL}

Na medida em que nos apropriamos do contexto e pontuamos as necessidades formativas a partir da ação de intervenção sistematizada, estruturamos e oferecemos ao Centro de Reabilitação o curso de Formação de Educadores "Formação de Educadores de Pessoas com Deficiência em Timor-Leste: noções teóricas e práticas interventivas”. O curso agregou elementos teóricos (conceituais) acerca do desenvolvimento humano e os diferentes tipos de deficiências, além da construção coletiva de planejamentos e construção de atividades.

O grupo de formandos foi composto por cinco pessoas e o trabalho de formação foi desenvolvido semanalmente, envolvendo essencialmente discussões dos conceitos teóricos sobre a deficiência e práticas pedagógicas, além da instrumentalização com exercícios e práticas para replicação. O curso teve duração de dois meses e os temas trabalhados foram: Educação x Educação Especial, Desenvolvimento Humano, Deficiência Física, Deficiência Sensorial, Deficiência Intelectual e, por último, Avaliação e Planejamento. Cada encontro Conexões: revista da Faculdade de Educação Física da UNICAMP, Campinas, v. 13, n. 4, p. 178-187, out./dez. 2015. ISSN: 1983-9030. 
foi organizado a partir de quatro momentos distintos: primeiro realizou-se a leitura e discussão dos registros (portfólio) individual dos formandos; posteriormente, realizávamos a discussão de conceitos importantes dentro do campo da educação especial e da deficiência, seguido de uma atividade prática correspondente ao tema; por fim, realizamos coletivamente o planejamento para o atendimento no Centro de Reabilitação.

\section{CONSIDERAÇÕES FINAIS}

A análise deste relato de experiência aponta caminhos promissores e desafiadores concomitantemente. Acerca do atendimento especializado na área da Educação Física, embora em um curto período de tempo, observam-se algumas mudanças no comportamento tanto das crianças quanto das mães. Inicialmente, era comum as mães, durante a realização das intervenções, tomarem a iniciativa e, muitas vezes, “fazer” o movimento pela/com a criança, agora, identifica-se uma postura mais ativa das crianças no denvolvimento das atividades. Se nas primeiras semanas, por exemplo, uma das mães quase não tirava a filha do seu colo, percebe-se agora que, além de deixá-la livre no chão, passou a retirar o aparelho das pernas da criança, antes do início do atendimento, para que a mesma tivesse mais mobilidade durante as atividades.

As atividades realizadas em grupo foram fundamentais não apenas para o desenvolvimento das crianças por meio da interação, mas também para a conscientização das mães sobre a capacidade de seus filhos agirem coletivamente, brincarem e se divertirem como qualquer outra criança. Destaca-se ainda que, durante as atividades de jogos coletivos, de forma natural, as crianças e jovens com deficiências passaram a se organizar em grupos e/ou equipes. Muitas vezes, durante as atividades com bola, os educadores timorenses organizaram estratégias para garantir a participação de todos na atividade. Podemos afirmar que, tanto nos momentos de intervenção pedagógica quanto no espaço do curso de formação dos educadores timorenses, é visível a mudança na forma como o grupo passou a interagir. Em diversos momentos, as crianças e jovens com deficiência, de forma autônoma, colaboraram com os colegas no processo de construção dos materiais ou na realização das atividades.

Conexões: revista da Faculdade de Educação Física da UNICAMP, Campinas, v. 13, n. 4, p. 178-187, out./dez. 2015. ISSN: 1983-9030. 
Acerca do desenvolvimento do curso de formação de educadores, destaca-se que a metodologia de registro em portfólio e o planejamento coletivo de atividades para posterior replicação foram fundamentais no desenvolvimento da formação. Esta metodologia garantiu, semanalmente, a avaliação processual da assimilação dos formandos a partir da leitura dos portfólios. Além disso, a participação nos planejamentos gerou nos formandos um sentimento de segurança e eles, naturalmente, se sentiam confiantes para condução das atividades nos momentos de intervenção com todo o grupo.

Entende-se a autonomia como princípio nos processos formativos, sobretudo por se tratar de um programa de cooperação internacional. De acordo com Soares ${ }^{6}$ é fundamental construir nos espaços de formação de educadores situações em que os formandos timorenses, coletivamente, reflitam e solucionem aquelas situações que não estão listadas em manuais, a partir de técnicas específicas, mas são, por outro lado, incertas, inesperadas, singulares, etc., ou seja, não são resolvidas a partir de um repertório de conhecimentos que já se possui, necessitando assim de uma postura autônoma frente ao desafio e uma reflexão sobre a ação realizada.

Entende-se que a cooperação brasileira, assim como as demais, deve buscar em suas práticas formativas: problematizar, refletir e encontrar coletivamente (com os pares timorenses) possíveis soluções para as questões que envolvem o desenvolvimento da educação no país, ou seja, fazer junto e não fazer por “alguém”. Os espaços não devem ser ocupados, mas sim partilhados.

\section{REFERÊNCIAS}

${ }^{1}$ TIMOR-LESTE. Ministério da Educação. Lei de Bases da Educação, 2008. Disponível em: <http://www.jornal.gov.tl/?q=node/876 >. Acesso em: 10 jun. 2014.

${ }^{2}$ COORDENAÇÃO DE APERFEIÇOAMENTO DE PESSOAL DE NÍVEL SUPERIOR (CAPES). Projeto Político Pedagógico do Programa de Qualificação de Docentes e Ensino de Língua Portuguesa no Timor-Leste - PQLP. Disponível em:

Conexões: revista da Faculdade de Educação Física da UNICAMP, Campinas, v. 13, n. 4, p. 178-187, out./dez. 2015. ISSN: 1983-9030. 
<http://pqlp.pro.br/pagina-exemplo/projeto-politico-pedagogico/>. Acesso em: 01 set. 2015.

${ }^{3}$ SASSAKI. R. K. Inclusão: construindo uma sociedade para todos. Rio de Janeiro: WVA,1997.

${ }^{4}$ LE BOULCH, J. A educação pelo movimento: a psicocinetica na idade escolar. Porto Alegre: Artes Médicas, 1983.

${ }^{5} \mathrm{CHICON}$, J. F. Prática psicopedagógica em crianças com necessidades educativas especiais: abordagem psicomotora. Vitória: CEFD/UFES, 1999.

${ }^{6}$ SOARES, F. R. Entre o querer e o fazer em formação contínua de professores/as: discursos e práticas em uma rede municipal de educação. 2012. 135 f. Dissertação (Mestrado em Ciências da Educação) - Faculdade de Psicologia e Ciências da Educação, Universidade do Porto, Portugal, 2012.

Recebido em: 17 nov. 2015

Aceito em:16 out. 2015

Contato: fran_rossetto@hotmail.com

Conexões: revista da Faculdade de Educação Física da UNICAMP, Campinas, v. 13, n. 4, p. 178-187, out./dez. 2015. ISSN: 1983-9030. 